\title{
Who clerks-in medical patients?
}

\author{
Authors: C Watters, ${ }^{1}$ R Osanlou, ${ }^{1}$ O Osanlou, ${ }^{1,2}$ K Clark $^{1}$ and C Constable ${ }^{1}$
}

\section{Aims}

To review the proportion of acute medical admissions clerked in by doctors of various levels and to suggest how to improve the efficiency of the medical take.

\section{Methods}

The chief registrar led this project at Warrington Hospital. At any one time, the acute team consists of 1 higher medical trainee, between 1-2 core medical trainees (CMTs), and 2-5 other doctors (GP trainees [GPSTs], foundation year [FY] doctors or staff grades). There is significant burden and responsibility placed on the lone higher medical trainee.

1100 notes were reviewed at random over an 11 month period to see who had clerked them in.

\section{Results}

Of the 1100 notes pulled, 726 (66\%) were correctly identified as medical patients. The others were mistakenly coded or incorrectly allocated as being under the care of the division of medicine.

It was possible to identify the person and grade of who first clerked the patient in 597/726 (82\%) of cases. Of these:

$><1 \%$ were clerked by the Foundation year 1 doctors

$>54 \%$ were clerked by FY2/GPST/SG/CMT level doctors

$>39 \%$ of all admissions were clerked by the higher medical trainee

$>6 \%$ were seen directly by a consultant

\section{Conclusions}

It is encouraging to see more patients seen directly by consultants than has been the case previously. It is disappointing to see that FY1 doctors are not actively engaged with clerking patients in and this seems a missed educational opportunity. Considering that there is only one higher medical trainee at any one time out of a team of up to 8 doctors, a considerable proportion of new admissions are clerked in by them.

Authors: ${ }^{1}$ Warrington and Halton NHS Foundation Trust;

${ }^{2}$ University of Liverpool
More junior trainees and consultants need to be involved in clerking patients to allow the higher medical trainees to fulfil their other responsibilities.

\section{Conflict of interest statement}

None. 